\section{Arneth-Index der neutrophilen Granulozyten}

\author{
A. M. Gressner ${ }^{1}$ und O. A. Gressner ${ }^{2}$ \\ ${ }^{1}$ Labor Dr. Wisplinghoff Berlin, Berlin, Deutschland \\ ${ }^{2}$ Labor Dr.Wisplinghoff Köln, Köln, Deutschland
}

Synonym(e) Arneth-Index; Arneth-Zahl

Englischer Begriff Arneth's count

Definition Heute nicht mehr gebräuchliche quantitative Subdifferenzierung der polymorphkernigen neutrophilen Granulozyten auf Basis der Zahl ihrer Kernsegmente $(n=1-5)$.

Beschreibung Der von dem deutschen Hämatologen J. Arneth (1873-1955) entwickelte Index gibt die prozentuale
Verteilung der nach Zahl ihrer Kernsegmente subklassifizierten neutrophilen Granulozyten ( $\vee$ Granulozyten, segmentkernige) an. Die übliche Zahl der Kernsegmente reifer polymorphkerniger Granulozyten variiert zwischen 2 (35\%) und 3 (41\%). Eine Abweichung nach unten (Linksverschiebung) kann u. a. bei Infektionen, Intoxikationen, Malignomen, Hämolyse, eine Abweichung nach oben (Rechtsverschiebung) u. a. bei Mangelversorgung mit $>$ Vitamin B12 und $\vee$ Folsäure und bei überalterten Neutrophilen beobachtet werden ( $\triangleright$ Granulozytopoese). Der Index hat heute keinen diagnostischen Stellenwert mehr.

\section{Literatur}

Arneth J (1904) Die neutrophilen weissen Blutkörperchen bei Infektions-Krankheiten. Fischer-Verlag, Jena 